\title{
SYMPLECTIC HYPERSURFACES IN THE COMPLEMENT OF AN ISOTROPIC SUBMANIFOLD
}

\author{
DENIS AUROUX, DAMIEN GAYET, AND JEAN-PAUL MOHSEN
}

\begin{abstract}
Using Donaldson's approximately holomorphic techniques, we construct symplectic hypersurfaces lying in the complement of any given compact isotropic submanifold of a compact symplectic manifold. We discuss the connection with rational convexity results in the Kähler case and various applications.
\end{abstract}

\section{INTRODUCTION}

It was first observed by Duval (see e.g. [Du]) that, in Kähler geometry, the notions of isotropy and rational convexity are tightly related to each other. Recall that a compact subset $N$ of $\mathbb{C}^{n}$ or more generally of a complex algebraic manifold is said to be rationally convex if there exists a complex algebraic hypersurface passing through any given point in the complement of $N$ and avoiding $N$. Among the results motivating the interest in this notion, one can mention the classical theorem of Oka and Weil (further improved by subsequent work) stating that every holomorphic function over a neighborhood of a rationally convex compact subset $N \subset \mathbb{C}^{n}$ can be uniformly approximated over $N$ by rational functions.

It was shown in 1995 by Duval and Sibony that, if a smooth compact submanifold of $\mathbb{C}^{n}$ is isotropic with respect to some Kähler structure on $\mathbb{C}^{n}$, then it is rationally convex [DS]. This result was extended in 1999 by Guedj to the context of complex projective manifolds :

Theorem 1 (Guedj $[\mathrm{Gu}])$. Let $(X, \omega, J)$ be a closed Kähler manifold, such that the cohomology class $\frac{1}{2 \pi}[\omega] \in H^{2}(X, \mathbb{R})$ is integral. Then any smooth compact isotropic submanifold $\mathcal{L} \subset X$ (possibly with boundary) is rationally convex, i.e. there exist complex hypersurfaces in $X$ passing through any given point in the complement of $\mathcal{L}$ and avoiding $\mathcal{L}$.

Because the concept of isotropic submanifold originates in symplectic geometry, it is natural to seek an analogue of this result for symplectic manifolds. Although the lack of an integrable almost-complex structure prevents the existence of holomorphic hypersurfaces in a general symplectic manifold, a suitable analogue may be found in Donaldson's construction of approximately holomorphic symplectic hypersurfaces.

Let $(X, \omega)$ be a closed compact symplectic manifold of real dimension $2 n$. Unless otherwise stated, we will always assume that the cohomology class $\frac{1}{2 \pi}[\omega] \in H^{2}(X, \mathbb{R})$ is integral ; this does not restrict the diffeomorphism type of $X$ in any way. A compatible almost-complex structure $J$ on $X$ and the corresponding Riemannian metric $g$ are also fixed. 
Let $L$ be a complex line bundle on $X$ with first Chern class $c_{1}(L)=\frac{1}{2 \pi}[\omega]$, endowed with a Hermitian structure and a Hermitian connection $\nabla^{L}$ whose curvature 2 -form is $-i \omega$. It was shown by Donaldson in [D1] that, when the integer $k$ is large enough, the line bundles $L^{\otimes k}$ admit many approximately $J$-holomorphic sections, some of which possess remarkable transversality properties ensuring that their zero sets are smooth symplectic submanifolds in $X$. Many interesting constructions in symplectic topology have recently been obtained by using the same techniques (see e.g. [A2], [D2] and $[\mathrm{S}]$ ).

Let us recall the following definitions. The almost-complex structure $J$ and the Hermitian connection on $L^{\otimes k}$ induced by that on $L$ yield $\partial$ and $\bar{\partial}$ operators on $L^{\otimes k}$. Since the connection on $L^{\otimes k}$ has curvature $-i k \omega$, we introduce the rescaled metric $g_{k}=k g$ on $X$, in order to be able to consider uniform bounds for covariant derivatives of sections of $L^{\otimes k}$. As a consequence of this rescaling, the diameter of $X$ is multiplied by $k^{1 / 2}$, and all derivatives of order $p$ are divided by $k^{p / 2}$.

Definition 1. Let $\left(s_{k}\right)_{k \gg 0}$ be a sequence of sections of $L^{\otimes k}$ over $X$. The sections $s_{k}$ are said to be asymptotically holomorphic if there exists a constant $C>0$ such that, for all $k$ and at every point of $X,\left|s_{k}\right|+\left|\nabla s_{k}\right|+$ $\left|\nabla \nabla s_{k}\right| \leq C$ and $\left|\bar{\partial} s_{k}\right|+\left|\nabla \bar{\partial} s_{k}\right| \leq C k^{-1 / 2}$, where the norms of the derivatives are evaluated with respect to the metrics $g_{k}=k \mathrm{~g}$.

The sections $s_{k}$ are said to be uniformly transverse to 0 if there exists a constant $\eta>0$ (independent of $k$ ) such that the sections $s_{k}$ are $\eta$-transverse to 0 , i.e. such that, for any $k$ and at any point $x \in X$ where $\left|s_{k}(x)\right|<\eta$, the covariant derivative $\nabla s_{k}(x): T_{x} X \rightarrow L_{x}^{\otimes k}$ is surjective and satisfies the bound $\left|\nabla s_{k}(x)\right|_{g_{k}}>\eta$.

With these definitions, Donaldson's construction amounts to showing the existence of a sequence of sections $s_{k}$ of $L^{\otimes k}$ which are at the same time asymptotically holomorphic and uniformly transverse to 0 [D1]. It then follows easily from these properties that, for large enough $k$, the zero sets $W_{k}$ of $s_{k}$ are smooth symplectic hypersurfaces in $X$.

Let $\mathcal{L}$ be a compact isotropic submanifold in $X$, not necessarily connected : we wish to show that one can get the symplectic hypersurfaces $W_{k}$ to lie in $X-\mathcal{L}$. The fundamental reason why it is reasonable to expect such a result is that, since $\omega$ vanishes over $\mathcal{L}$, the line bundle $L_{\mid \mathcal{L}}$ comes equipped with a flat connection. However $L^{\otimes k}$ admits non-vanishing sections over $\mathcal{L}$ only when its restriction to $\mathcal{L}$ is topologically trivial ; if $\mathcal{L}$ is not simply connected, this can restrict the admissible values of the parameter $k$. For example, if $X=\mathbb{C P}^{2}$ and $\mathcal{L}=\mathbb{R P}^{2}$, an easy calculation in homology with $\mathbb{Z} / 2$ coefficients shows that any symplectic submanifold of odd degree must intersect $\mathcal{L}$. Our main result is the following :

Theorem 2. Let $\mathcal{L}$ be a compact isotropic submanifold in $X$, and let $N$ be the order of the torsion part of $H_{1}(\mathcal{L}, \mathbb{Z})$. Then, for all large enough values of $k$, there exist asymptotically holomorphic sections $s_{k}$ of $L^{\otimes k}$ over $X$ whose zero sets $W_{k}$ are smooth symplectic submanifolds, disjoint from $\mathcal{L}$ whenever $k$ is a multiple of $N$. Moreover, $W_{k}$ can be assumed to pass through any given point $x_{0} \in X-\mathcal{L}$. 
This result is mildly surprising when one considers the results obtained in [D1] and [A1] indicating that, when $k$ increases, the submanifolds $W_{k}$ tend to fill all of $X$. There is no contradiction, though, as the distance by which the submanifolds $W_{k}$ given by Theorem 2 stay away from $\mathcal{L}$ actually decreases like $k^{-1 / 2}$.

Remark 1. (a) Theorem 2 remains valid when $\mathcal{L}$ has non-empty boundary; see $[\mathrm{M}]$ for details.

(b) When $X$ is a Kähler manifold, one can perform the construction in such a way that the sections $s_{k}$ are holomorphic. The submanifolds $W_{k}$ are then complex hypersurfaces ; this provides a new proof of Guedj's rational convexity result.

(c) When the cohomology class $\frac{1}{2 \pi}[\omega]$ is no longer assumed to be integral, the line bundle $L$ is no longer defined, but it is still possible to obtain symplectic hypersurfaces in $X$ which avoid the submanifold $\mathcal{L}$ and pass through any given point in $X-\mathcal{L}$.

Additional motivation for these results can be found in the work of Biran [B], where the notion of Lagrange skeleton of a symplectic manifold of Kähler type with respect to a hypersurface of Donaldson type is defined. As will be explained in $\S 3$, Theorem 2 can be interpreted in this context as a flexibility result for Lagrange skeleta in large degrees.

More importantly, it was observed by Seidel and Viterbo that Theorem 2 implies that if $\mathcal{L}$ is Lagrangian then its homology class is a primitive element of $H_{n}\left(X-W_{k}\right)$ (see $\left.\S 3\right)$; this remark might lead to obstructions to the existence of certain Lagrangian embeddings.

Note. Different proofs of Theorem 2 were obtained independently by the three authors ; the curious reader is referred to $[\mathrm{M}]$ and $[\mathrm{Ga}]$ for various alternate arguments and generalizations.

The authors wish to thank Claude Viterbo, Paul Seidel and Paul Biran for motivating discussions and for suggesting applications of Theorem 2 . The authors are respectively thankful to Ivan Smith, Julien Duval, Bruno Sévennec and Emmanuel Giroux for discussions and advice.

\section{Proof of Theorem 2}

We first define the notion of concentrated sections of $L^{\otimes k}$ :

Definition 2. Asymptotically holomorphic sections $s_{k}$ of $L^{\otimes k}$ are said to be concentrated over a subset $N \subset X$ if there exist positive constants $\lambda, c$ and $C$ (independent of $k$ ) such that for all $y \in N,\left|s_{k}(y)\right| \geq c$, and, for all $y \in X,\left|s_{k}(y)\right| \leq C \exp \left(-\lambda d(y, N)^{2}\right)$, where $d(.,$.$) is the distance induced$ by $g_{k}$. When the subset $N$ consists of a single point $x \in X$, we say that the sections $s_{k}$ are concentrated at $x$.

With this terminology, recall the following result (Proposition 11 of [D1]) :

Lemma 1 (Donaldson). For all large enough $k$ the line bundles $L^{\otimes k}$ admit asymptotically holomorphic sections $\sigma_{k, x}$ concentrated at any given point $x \in X$.

As the properties of the sections $\sigma_{k, x}$ play an important role in the argument, let us recall briefly their construction. 
Remember that, at any point $x \in X$, it is possible to find a local approximately holomorphic Darboux coordinate chart, i.e. a local symplectomorphism $\psi:(X, x, \omega) \rightarrow\left(\mathbb{C}^{n}, 0, \omega_{0}\right)$ such that, with respect to $J$ and the standard complex structure of $\mathbb{C}^{n}, \bar{\partial} \psi(x)=0$ and $|\nabla \bar{\partial} \psi|_{g}$ is bounded uniformly by a constant $C$. The compactness of $X$ implies that the size of the neighborhood over which $\psi$ is defined and the value of the constant $C$ can be assumed not to depend on the chosen point $x$.

In our case, we will moreover require that, whenever the point $x$ belongs to the given isotropic submanifold $\mathcal{L}$, the coordinate map $\psi$ locally sends $\mathcal{L}$ to a linear subspace in $\mathbb{C}^{n}$ (obviously isotropic). The existence of Darboux coordinate charts with this property is a very classical result of Weinstein ([W], see also $[\mathrm{McS}])$; it is an immediate observation that the coordinate map can still be chosen to satisfy $\bar{\partial} \psi(x)=0$, and the compactness of $\mathcal{L}$ implies the existence of uniform estimates on $|\nabla \bar{\partial} \psi|$ and on the size of the coordinate chart.

In a Darboux coordinate chart, a suitable unitary gauge transformation leads to a local trivialization of $L^{\otimes k}$ in which the connection 1-form is given by $\frac{k}{4} \sum\left(z_{j} d \bar{z}_{j}-\bar{z}_{j} d z_{j}\right)$. The local section defined by $f_{k}(z)=\exp \left(-k|z|^{2} / 4\right)$ is then holomorphic over a neighborhood of 0 in $\mathbb{C}^{n}$. Pulling back $f_{k}$ via the coordinate chart $\psi$, one obtains sections $\hat{\sigma}_{k, x}$ of $L^{\otimes k}$ over a neighborhood of $x$ in $X$, and it easily follows from the estimates on $\bar{\partial} \psi$ that these sections are asymptotically holomorphic.

Finally, multiplying $\hat{\sigma}_{k, x}$ by a smooth cut-off function vanishing at distance $k^{-1 / 6}$ from $x$ yields the desired asymptotically holomorphic sections $\sigma_{k, x}$, easily shown to be concentrated at the point $x$ (see [D1]).

Recall from [D1] (see also [A1]) that asymptotically holomorphic sections with uniform transversality estimates are constructed by an iterative process, where one starts with any given asymptotically holomorphic sections $s_{k}$ of $L^{\otimes k}$ (e.g. $s_{k}=0$ ) and perturbs them over small open subsets of $X$ in order to achieve transversality over those subsets ; successive smaller and smaller perturbations are performed in such a way that the transversality property gained at each step is preserved by all subsequent perturbations, until transversality holds over all of $X$. In particular, given any constant $C>0$ it is possible to ensure that the constructed sections $\tilde{s}_{k}$ differ from the given sections $s_{k}$ by less than $C$ in $C^{1}$ norm (i.e., at every point of $X$ we have $\left.\left|\tilde{s}_{k}-s_{k}\right|+\left|\nabla \tilde{s}_{k}-\nabla s_{k}\right|_{g_{k}} \leq C\right)[\mathrm{A} 1]$.

Therefore, in order to prove Theorem 2 (without requiring yet the submanifolds to pass through a given point of $X-\mathcal{L})$, it is sufficient to construct asymptotically holomorphic sections $\sigma_{k, \mathcal{L}}$ of $L^{\otimes k}$, concentrated over $\mathcal{L}$ for $k$ ranging over all large enough multiples of $N=\mid$ Tor $H_{1}(\mathcal{L}, \mathbb{Z}) \mid$. By definition these sections satisfy a uniform lower bound over $\mathcal{L}$ by some constant $c>0$, and perturbing them by less than $c / 2$ we get (for large enough $k$ ) uniformly transverse sections which do not vanish over $\mathcal{L}$. Our next ingredient is the following observation :

Lemma 2. Given any compact isotropic submanifold $\mathcal{L} \subset X$, there exists a constant $C_{\mathcal{L}}>0$ such that, whenever $k$ is a multiple of $N=\left|\operatorname{Tor} H_{1}(\mathcal{L}, \mathbb{Z})\right|$, the restriction of $L^{\otimes k}$ to $\mathcal{L}$ admits a section $\tau_{k}$ such that $\left|\tau_{k}(x)\right|=1$ and $\left|\nabla \tau_{k}(x)\right|_{g} \leq C_{\mathcal{L}}$, i.e. $\left|\nabla \tau_{k}(x)\right|_{g_{k}} \leq C_{\mathcal{L}} k^{-1 / 2}$, at every point $x \in \mathcal{L}$. 
Proof. Since $\mathcal{L}$ is isotropic, the restriction to $\mathcal{L}$ of the connection $\nabla^{L}$ on $L$ is flat ; therefore the first Chern class $c_{1}\left(L_{\mid \mathcal{L}}\right)$, although not necessarily trivial, belongs to the kernel of the natural map $\iota: H^{2}(\mathcal{L}, \mathbb{Z}) \rightarrow H^{2}(\mathcal{L}, \mathbb{R})$. By the universal coefficients theorem (see e.g. [BT], page 194), $\operatorname{Ker}(\iota)=$ Tor $H^{2}(\mathcal{L}, \mathbb{Z}) \simeq$ Tor $H_{1}(\mathcal{L}, \mathbb{Z})$. It follows that the order of $c_{1}\left(L_{\mid \mathcal{L}}\right)$ divides $N$, so that the complex line bundle $L_{\mid \mathcal{L}}^{\otimes k}$ has zero first Chern class and hence is topologically trivial whenever $k$ is a multiple of $N$.

Fix a trivialization of $L^{\otimes k}$ over $\mathcal{L}$, and consider the 1-form $\alpha_{k} \in \Omega^{1}(\mathcal{L}, i \mathbb{R})$ representing the connection on $L^{\otimes k}$ induced by $\nabla^{L}$. We work with the metric on $\mathcal{L}$ induced by $g$, and observe that a suitable choice of trivialization of $L^{\otimes k}$ ensures that the 1-form $\alpha_{k}$ and its derivatives satisfy uniform bounds which depend only on the geometry of $\mathcal{L}$ and not on $k$.

Indeed, it is well-known that the moduli space of flat unitary connections on the trivial complex line bundle over $\mathcal{L}$ up to $U(1)$ gauge transformations is compact and isomorphic to $H^{1}(\mathcal{L}, \mathbb{R}) / H^{1}(\mathcal{L}, \mathbb{Z})$. Therefore, a well-chosen gauge transformation makes it possible to obtain uniform bounds on the 1 -form $\alpha_{k}$ and its derivatives, independently of $k$. More precisely, a first gauge transformation in the identity component can be used to make the closed 1-form $\alpha_{k}$ harmonic, while the flexibility coming from the connected components of the gauge group makes it possible to ensure that $\alpha_{k}$ lies in a fixed bounded subset of $H^{1}(\mathcal{L}, \mathbb{R})$.

Let $\tau_{k}$ be the section of $L^{\otimes k}$ over $\mathcal{L}$ which identifies with the constant function 1 in the chosen trivialization : clearly, $\left|\tau_{k}\right|=1$ at every point of $\mathcal{L}$ and the derivatives of $\tau_{k}$ are bounded by uniform constants independently of $k$ with respect to the metric $g$.

Remark. The bounds satisfied by $\alpha_{k}$ and $\nabla \tau_{k}$ depend on the minimum $g$ length $\delta(\mathcal{L})$ of a homotopically non-trivial loop in $\mathcal{L}$; in fact $C_{\mathcal{L}}$ must be at least of the order of $\delta(\mathcal{L})^{-1}$. This is one of the reasons why the submanifold $\mathcal{L}$ cannot be allowed to vary with $k$, another one being that we need to control the size of the balls centered at points of $\mathcal{L}$ which can be trivialized by Weinstein's theorem.

Throughout the remainder of this section we assume that $k$ is a multiple of $N$. For each such $k$, let $P_{k}$ be a finite set of points of $\mathcal{L}$ such that the balls of $g_{k}$-radius 1 centered at the points of $P_{k}$ cover $\mathcal{L}$ and any two points of $P_{k}$ are at $g_{k}$-distance at least $\frac{2}{3}$ from each other. Such a set can be constructed by covering $\mathcal{L}$ by finitely many balls of $g_{k}$-radius $\frac{1}{3}$ and iteratedly removing the points that are too close to each other (see also [D1]).

Define the sections

$$
\sigma_{k, \mathcal{L}}=\sum_{p \in P_{k}} \frac{\tau_{k}(p)}{\sigma_{k, p}(p)} \sigma_{k, p}
$$

of $L^{\otimes k}$ over $X$. The sections $\sigma_{k, \mathcal{L}}$ are linear combinations of the asymptotically holomorphic sections $\sigma_{k, p}$, with coefficients unitary complex numbers (recall that $\left|\tau_{k}(p)\right|=\left|\sigma_{k, p}(p)\right|=1$ ). Therefore, because any two points of $P_{k}$ are mutually $g_{k}$-distant of at least $\frac{2}{3}$ and because the sections $\sigma_{k, p}$ are concentrated at points, a standard argument ([D1],[S]) shows that the sections $\sigma_{k, \mathcal{L}}$ are uniformly bounded and asymptotically holomorphic. 
We now show that the sections $\sigma_{k, \mathcal{L}}$ are concentrated over $\mathcal{L}$. The decay properties of $\sigma_{k, \mathcal{L}}$ away from $\mathcal{L}$ follow from the following lemma :

Lemma 3. Let $P_{k} \subset X$ be a finite set of points whose mutual $g_{k}$-distance is bounded from below by a constant $\delta>0$. Let $\left(\alpha_{k, p}\right)_{p \in P_{k}}$ be a family of complex numbers such that $\left|\alpha_{k, p}\right| \leq 1 \forall p \in P_{k}$, and let $s_{k}=\sum_{p \in P_{k}} \alpha_{k, p} \sigma_{k, p}$. Then there exist constants $C_{\delta}$ and $\lambda_{\delta}$, independent of $k$ and $P_{k}$, such that $\left|s_{k}(x)\right| \leq C_{\delta} \exp \left(-\lambda_{\delta} d_{g_{k}}\left(x, P_{k}\right)^{2}\right)$ at every point of $X$.

Proof. Because $\sigma_{k, p}$ is supported in $B_{g}\left(p, 2 k^{-1 / 6}\right)$, we can restrict ourselves to only considering points in a fixed ball around the given point $x \in X$; since the $g_{k}$-distance between any two points of $P_{k}$ is greater than $\delta$, this implies that the number of points $p \in P_{k}$ lying within a given fixed $g_{k}$-distance $\rho$ of $x$ is bounded by $Q(\rho)$, where $Q$ is a polynomial depending only on $\delta$. Therefore, using the existence of a bound $\left|\sigma_{k, p}(x)\right| \leq C^{\prime} \exp \left(-\lambda^{\prime} d(x, p)^{2}\right)$ for $\sigma_{k, p}$ and ordering the points of $P_{k}$ according to their distance from $x$, we get the desired bound on $\left|s_{k}(x)\right|$ by summing over concentric slices.

We immediately conclude that $\left|\sigma_{k, \mathcal{L}}(x)\right| \leq C_{2 / 3} \exp \left(-\lambda_{2 / 3} d_{g_{k}}(x, \mathcal{L})^{2}\right)$. It remains to be shown that the norm of $\sigma_{k, \mathcal{L}}$ at a point of $\mathcal{L}$ admits a uniform lower bound. For this, we first prove the following result :

Lemma 4. If $k$ is large enough, and if $p$ and $x$ are two points of $\mathcal{L}$ such that $d_{g_{k}}(p, x) \leq k^{1 / 10}$, then $\sigma_{k, p}(x) \neq 0$ and

$$
\left|\arg \left(\frac{\sigma_{k, p}(x)}{\tau_{k}(x)}\right)-\arg \left(\frac{\sigma_{k, p}(p)}{\tau_{k}(p)}\right)\right| \leq \frac{\pi}{4} .
$$

Proof. Since the $g$-distance between $x$ and $p$ is less than $k^{-2 / 5}$, the cut-off function used to define $\sigma_{k, p}$ is equal to 1 at $x$, and therefore $\sigma_{k, p}(x) \neq 0$.

We work in the same local coordinate chart $\psi$ and local trivialization of $L^{\otimes k}$ that were used to define $\sigma_{k, p}$; we write $\psi(x)=u$, and consider the radial path $\gamma(t)=\psi^{-1}(t u)$ from $p$ to $x$. Recall that the connection on $L^{\otimes k}$ is expressed as $d+A_{k}=d+\frac{k}{4} \sum\left(z_{j} d \bar{z}_{j}-\bar{z}_{j} d z_{j}\right)$, while $\sigma_{k, p}$ is locally given by the function $\exp \left(-\frac{k}{4}|z|^{2}\right)$. Therefore one easily checks that

$$
\int_{0}^{1}\left(\frac{\nabla \sigma_{k, p}}{\sigma_{k, p}}\right)_{\gamma(t)} \cdot \gamma^{\prime}(t) d t=\int_{0}^{1} d\left(-\frac{k}{4}|z|^{2}\right)_{(t u)} \cdot u d t=-\frac{k}{4}|u|^{2} \in \mathbb{R} .
$$

Recall that by construction we require that $\psi$ locally maps $\mathcal{L}$ to a linear subspace of $\mathbb{C}^{n}$. Therefore the radial path $\gamma$ is contained in $\mathcal{L}$, and we can use the bound on $\nabla \tau_{k}$ given by Lemma 2 to obtain that

$$
\left|\int_{0}^{1}\left(\frac{\nabla \tau_{k}}{\tau_{k}}\right)_{\gamma(t)} \cdot \gamma^{\prime}(t) d t\right| \leq \int_{0}^{1}\left|\left(\nabla \tau_{k}\right)_{\gamma(t)}\right| \cdot\left|\gamma^{\prime}(t)\right| d t=O\left(k^{-2 / 5}\right) .
$$

Therefore,

$$
\arg \left(\frac{\sigma_{k, p}(x)}{\tau_{k}(x)}\right)-\arg \left(\frac{\sigma_{k, p}(p)}{\tau_{k}(p)}\right)=\operatorname{Im}\left[\int_{0}^{1}\left(\frac{\nabla \sigma_{k, p}}{\sigma_{k, p}}-\frac{\nabla \tau_{k}}{\tau_{k}}\right)_{\gamma(t)} \cdot \gamma^{\prime}(t) d t\right]
$$

is bounded by a constant times $k^{-2 / 5}$, which gives the result. 
Lemma 4 implies the existence of a uniform lower bound on $\sigma_{k, \mathcal{L}}$ at any point of $\mathcal{L}$. Indeed, consider a point $x \in \mathcal{L}$, and let $p$ be the point of $P_{k}$ closest to $x$. By construction $d_{g_{k}}(x, p) \leq 1$, and therefore there exists a constant $c>0$ (independent of $x, p$ and $k$ ) such that $\left|\sigma_{k, p}(x)\right| \geq c$. By Lemma 4 we know that the contributions of the various points $q \in P_{k}$ whose $g_{k}$-distance to $x$ is less than $k^{1 / 10}$ cannot cancel each other, and we have

$$
\left|\sum_{\substack{q \in P_{k} \\ d(x, q) \leq k^{1 / 10}}} \frac{\tau_{k}(q)}{\sigma_{k, q}(q)} \sigma_{k, q}(x)\right| \geq\left|\sigma_{k, p}(x)\right| \geq c .
$$

On the other hand, Lemma 3 implies that the contribution of the remaining points of $P_{k}$ decreases exponentially with $k$. Therefore, when $k$ is large enough we get that $\left|\sigma_{k, \mathcal{L}}(x)\right| \geq c / 2$ at any point $x$ of $\mathcal{L}$; in fact, we even get that $\sup _{x \in \mathcal{L}}\left|\arg \left(\sigma_{k, \mathcal{L}}(x) / \tau_{k}(x)\right)\right|$ becomes arbitrarily small for large $k$.

We conclude that the asymptotically holomorphic sections $\sigma_{k, \mathcal{L}}$ are concentrated over $\mathcal{L}$, which ends the argument : perturbing $\sigma_{k, \mathcal{L}}$ by less than $c / 4$ we obtain asymptotically holomorphic sections $\tilde{\sigma}_{k, \mathcal{L}}$ satisfying a uniform transversality property, and by construction their zero sets are (asymptotically holomorphic) symplectic submanifolds which do not intersect $\mathcal{L}$.

The final step to complete the proof of Theorem 2 is to show that these asymptotically holomorphic hypersurfaces can be made to pass through a given point $x_{0} \in X-\mathcal{L}$. Considering the sections $u_{k, x_{0}}=k^{1 / 2} z_{1} \sigma_{k, x_{0}}$, where $z_{1}$ is a local approximately holomorphic coordinate function at $x_{0}$, the idea is to work with $\sigma_{k, \mathcal{L}}+u_{k, x_{0}}$ instead of $\sigma_{k, \mathcal{L}}$. Indeed, observing that for large $k$ the support of $u_{k, x_{0}}$ is disjoint from $\mathcal{L}$, a small perturbation of $\sigma_{k, \mathcal{L}}+u_{k, x_{0}}$ yields asymptotically holomorphic hypersurfaces $W_{k}$ avoiding $\mathcal{L}$ and passing through a point $x$ within unit $g_{k}$-distance of $x_{0}$. It is then possible to find a Hamiltonian diffeomorphism $\phi$ preserving $\mathcal{L}$, mapping $x$ to $x_{0}$, and sufficiently close to the identity in order to ensure the asymptotic holomorphicity of $\phi\left(W_{k}\right)$.

Remark. When $\mathcal{L}$ is Lagrangian, Theorem 2 can also be proved by arguing along the following lines. By Weinstein's Lagrangian neighborhood theorem, a neighborhood $V$ of $\mathcal{L}$ in $X$ is symplectomorphic to a neighborhood of the zero section in $T^{*} \mathcal{L}$ with its standard symplectic structure $d p \wedge d q$; the fibers of $\pi: T^{*} \mathcal{L} \rightarrow \mathcal{L}$ can be chosen $g$-orthogonal to $\mathcal{L}$ at every point of $\mathcal{L}$. Consider the trivialization of $L^{\otimes k}$ over $\mathcal{L}$ given by the section $\tau_{k}$ of Lemma 2, and extend it over $V$ in such a way that the connection 1-form is given by $\beta_{k}=\pi^{*} \alpha_{k}-i k p d q$, where $\alpha_{k}$ is the same 1 -form on $\mathcal{L}$ as in Lemma 2. It can then be checked that the sections of $L^{\otimes k}$ over $V$ defined by $s_{k}=\exp \left(-\frac{1}{2} k|p|_{g}^{2}\right)$ (where $|\cdot|_{g}$ is the metric induced by $g_{\mid \mathcal{L}}$ on the fibers of $\left.T^{*} \mathcal{L}\right)$ are asymptotically holomorphic ; multiplying $s_{k}$ by a suitable cut-off function we obtain asymptotically holomorphic sections concentrated over $\mathcal{L}$, from where Theorem 2 is easily obtained.

\section{REMARKS AND APPLICATIONS}

3.1. The Kähler case. We consider the case where $(X, \omega, J)$ is a Kähler manifold, and show how the construction can be performed in the holomorphic category (Remark $1(b)$ ) using the ideas of Donaldson (see pp. 
696-700 of [D1]). The first observation is that near any point $x \in X$ there exists a local holomorphic section of $L$ which, in the same local trivialization of $L$ as in the proof of Lemma 1 , is given by a function $f$ such that $f(z)=1-\frac{1}{4}|z|^{2}+O\left(|z|^{3}\right)$ and $d f(z)=-\frac{1}{4} \sum_{j}\left(z_{j} d \bar{z}_{j}+\bar{z}_{j} d z_{j}\right)+O\left(|z|^{2}\right)$; see the proof of Lemma 36 of [D1].

Multiplying $f(z)^{k}$ by a smooth cut-off function at distance $k^{-1 / 6}$ from $x$ yields asymptotically holomorphic sections $\sigma_{k, x}$ of $L^{\otimes k}$, concentrated at $x$ as in Lemma 1 ; moreover, as observed by Donaldson in [D1], there exist holomorphic sections $\tilde{\sigma}_{k, x}$ of $L^{\otimes k}$ such that $\sup \left|\tilde{\sigma}_{k, x}-\sigma_{k, x}\right| \leq C \exp \left(-a k^{1 / 3}\right)$, with $a$ and $C$ positive constants (independent of $k$ and $x$ ).

We now proceed as in $\S 2.1$, using the new sections $\sigma_{k, x}$ instead of those obtained in Lemma 1. The argument remains the same, the only difference being in the proof of Lemma 4 where the l.h.s. of (1) becomes equal to

$$
\int_{0}^{1} \frac{\left(d+A_{k}\right) f(z)_{(t u)}^{k}}{f(t u)^{k}} \cdot u d t=\int_{0}^{1} k\left(\frac{d f}{f}\right)_{(t u)} \cdot u d t=-\frac{k}{4}|u|^{2}+O\left(k|u|^{3}\right) .
$$

Since $|u|$ is at most of the order of $k^{-2 / 5}$ the imaginary part of this quantity is bounded by $O\left(k^{-1 / 5}\right)$, which is enough to prove Lemma 4 and hence construct $\sigma_{k, \mathcal{L}}$ as in $\S 2.1$.

Replacing $\sigma_{k, x}$ by $\tilde{\sigma}_{k, x}$ in the definition of $\sigma_{k, \mathcal{L}}$, we obtain holomorphic sections $\tilde{\sigma}_{k, \mathcal{L}}$ which differ from $\sigma_{k, \mathcal{L}}$ by at most $C \exp \left(-a k^{1 / 3}\right) \operatorname{card}\left(P_{k}\right)$ and therefore also satisfy a uniform lower bound over $\mathcal{L}$. It is then possible to conclude as usual, by adding a linear combination of the sections $\tilde{\sigma}_{k, x}$ to $\tilde{\sigma}_{k, \mathcal{L}}$ in order to achieve uniform transversality.

Alternately, given a point $x_{0} \in X-\mathcal{L}$, one can add a multiple of $\tilde{\sigma}_{k, x_{0}}$ to $\tilde{\sigma}_{k, \mathcal{L}}$ in order to obtain holomorphic sections $\tilde{\sigma}_{k, \mathcal{L}, x_{0}}$ which vanish at $x_{0}$ while remaining bounded away from zero over $\mathcal{L}$. In terms of the projective embeddings $i: X \rightarrow \mathbb{P} H^{0}\left(L^{\otimes k}\right)^{*}$, these sections correspond to hyperplanes passing through $i\left(x_{0}\right)$ while avoiding $i(\mathcal{L})$. A small generic perturbation yields a hyperplane passing through $i\left(x_{0}\right)$ which intersects $i(X)$ transversely and still avoids $i(\mathcal{L})$; this gives smooth complex hypersurfaces passing through $x_{0}$ and avoiding $\mathcal{L}$, giving a new proof of Guedj's result.

3.2. The non-integral case. In this section we no longer assume that the cohomology class $\frac{1}{2 \pi}[\omega]$ is integral, as in Remark $1(c)$. As in [D1] the idea is to perturb the symplectic form $\omega$ into a symplectic form $\omega^{\prime}$ such that $\frac{1}{2 \pi}\left[\omega^{\prime}\right]$ is proportional to an integral class, and work with a multiple of $\omega^{\prime}$. It is however necessary to ensure that $\mathcal{L}$ remains isotropic.

Because $\frac{1}{2 \pi}[\omega]$ lies in the kernel of the restriction map from $H^{2}(X, \mathbb{R})$ to $H^{2}(\mathcal{L}, \mathbb{R})$, it is the image of a class $\alpha \in H^{2}(X, \mathcal{L} ; \mathbb{R})$. Moreover, $H^{2}(X, \mathcal{L} ; \mathbb{Q})$ contains elements lying arbitrarily close to $\alpha$ in $H^{2}(X, \mathcal{L} ; \mathbb{R})$. Therefore, by adding to $\omega$ an arbitrarily small closed 2 -form vanishing over $\mathcal{L}$, we obtain a symplectic form $\omega^{\prime}$ such that $\frac{1}{2 \pi}\left[\omega^{\prime}\right]$ is the image of a class in $H^{2}(X, \mathcal{L} ; \mathbb{Q})$ and hence belongs to $H^{2}(X, \mathbb{Q})$. By construction, $\omega^{\prime}$ satisfies up to multiplication by a constant factor the required integrality condition, and $\mathcal{L}$ is $\omega^{\prime}$-isotropic.

The symplectic form $\omega^{\prime}$ admits a compatible almost-complex structure $J^{\prime}, C^{0}$-close to $J$; since $\omega\left(v, J^{\prime} v\right)>0 \forall v \in T X$, any $J^{\prime}$-complex subspace is $\omega$-symplectic. So, if a sequence of submanifolds $W_{k} \subset X$ is asymptotically 
$J^{\prime}$-holomorphic, then $W_{k}$ is a symplectic submanifold of $(X, \omega)$ for large enough $k$. One then concludes by applying Theorem 2 to $\left(X, \omega^{\prime}, J^{\prime}\right)$.

3.3. Uniqueness up to isotopy. It was shown in [A1] that the symplectic submanifolds constructed by Donaldson in [D1] are, for each large enough value of $k$, canonical up to symplectic isotopy, independently of the almostcomplex structure $J$. One may ask whether in our case the submanifolds $W_{k}$ are canonical up to a symplectic isotopy of $X$ preserving $\mathcal{L}$; such a uniqueness property does not hold in general, because the homotopy class of the non-vanishing section $s_{k}$ of $L^{\otimes k}$ over $\mathcal{L}$ plays a determining role.

Let $\gamma$ be a non-contractible loop in $\mathcal{L}$ bounding a disc $D$ in $X$ : the homotopy class of the non-vanishing section $\left(s_{k}\right)_{\mid \gamma}$ over $\gamma$ determines the number of zeroes of $s_{k}$ over $D$, i.e. the linking number of $W_{k}$ with $\gamma$, which can be modified by choosing different trivializations of $L^{\otimes k}$ over $\mathcal{L}$. Still, when $\mathcal{L}$ is simply connected the homotopy classes of the nowhere vanishing sections $\left(s_{k}\right)_{\mid \mathcal{L}}$ are uniquely determined.

Even though it seems reasonable to expect that the isotopy class of asymptotically holomorphic hypersurfaces in $X-\mathcal{L}$ should only depend on the homotopy class of $\left(s_{k}\right)_{\mid \mathcal{L}}$, our techniques do not allow us to prove so strong a statement ; we are only able to prove that the submanifolds constructed in $\S 2$ (using either the given proof or the alternate argument sketched at the end) are canonical up to symplectic isotopy in $X-\mathcal{L}$. For this, we use the control on the complex argument of $\left(s_{k}\right)_{\mid \mathcal{L}}$ given by the construction : it follows directly from Lemma 4 and the subsequent discussion that for large $k$ the argument of $s_{k} / \tau_{k}$ remains small at every point of $\mathcal{L}$.

Proposition 1. Let $\tau_{k}^{0}$ and $\tau_{k}^{1}$ be sections of $L^{\otimes k}$ over $\mathcal{L}$ belonging to the same homotopy class and such that $\left|\tau_{k}^{i}\right| \equiv 1$ and $\left|\nabla \tau_{k}^{i}\right|_{g}=O(1)$. Let $s_{k}^{0}$ and $s_{k}^{1}$ be asymptotically holomorphic sections of $L^{\otimes k}$ over $X$, uniformly transverse to 0 , uniformly bounded from below over $\mathcal{L}$, and such that the bound $\left|\arg \left(s_{k}^{i} / \tau_{k}^{i}\right)\right| \leq \frac{\pi}{3}$ holds at every point of $\mathcal{L}$. Then for large enough $k$ their zero sets $W_{k}^{0}$ and $W_{k}^{1}$ differ by a symplectic isotopy preserving $\mathcal{L}$.

Proof. We use the same one-parameter argument as in [A1] in order to construct for large $k$ a one-parameter family of asymptotically holomorphic sections $s_{k}^{t}$, bounded from below on $\mathcal{L}$, interpolating between $s_{k}^{0}$ and $s_{k}^{1}$. First, choosing a trivialization of $L^{\otimes k}$ over $\mathcal{L}$ to express $\tau_{k}^{i}$ in the form $\exp \left(\phi_{k}^{i}\right)$ for $i \in\{0,1\}$, we define sections $\tau_{k}^{t}$ of $L_{\mid \mathcal{L}}^{\otimes k}$ for $t \in[0,1]$ by $\tau_{k}^{t}=$ $\exp \left((1-t) \phi_{k}^{0}+t \phi_{k}^{1}\right)$. Observing that $\left|\tau_{k}^{t}\right| \equiv 1$ and $\left|\nabla \tau_{k}^{t}\right|_{g}=O(1)$ for all $t$, we can define sections $\sigma_{k, \mathcal{L}}^{t}=\sum_{p \in P_{k}}\left(\tau_{k}^{t}(p) / \sigma_{k, p}(p)\right) \sigma_{k, p}$ of $L^{\otimes k}$ over $X$ which are asymptotically holomorphic and concentrated over $\mathcal{L}$.

Define $s_{k}^{t}$ to be equal to $(1-3 t) s_{k}^{0}+3 t \sigma_{k, \mathcal{L}}^{0}$ for $t \in\left[0, \frac{1}{3}\right]$, to $\sigma_{k, \mathcal{L}}^{3 t-1}$ for $t \in\left[\frac{1}{3}, \frac{2}{3}\right]$ and to $(3-3 t) \sigma_{k, \mathcal{L}}^{1}+(3 t-2) s_{k}^{1}$ for $t \in\left[\frac{2}{3}, 1\right]$. All these sections are asymptotically holomorphic ; observing that for $i \in\{0,1\}$ the arguments of $s_{k}^{i}$ and $\sigma_{k, \mathcal{L}}^{i}$ both remain within $\frac{\pi}{3}$ of that of $\tau_{k}^{i}$ at every point of $\mathcal{L}$, they also satisfy a uniform lower bound by some constant $c>0$ at every point of $\mathcal{L}$.

Let $\gamma>0$ be the uniform transversality estimate satisfied by $s_{k}^{i}$ for $i \in\{0,1\}$. Applying the main theorem of [A1], we obtain, provided that 
$k$ is large enough, uniformly transverse sections $\tilde{s}_{k}^{t}$ of $L^{\otimes k}$ depending continuously on $t$ and differing from $s_{k}^{t}$ by at most $\frac{1}{2} \inf (c, \gamma)$ in $C^{1}$ norm ; slightly modifying this 1-parameter family near its extremities we can safely assume that $\tilde{s}_{k}^{0}=s_{k}^{0}$ and $\tilde{s}_{k}^{1}=s_{k}^{1}$ (see Corollary 2 in [A1]). The zero sets of $\tilde{s}_{k}^{t}$ are then symplectic hypersurfaces $W_{k}^{t} \subset X-\mathcal{L}$ realizing a smooth isotopy between $W_{k}^{0}$ and $W_{k}^{1}$. The argument in $\S 4.2$ of [A1] then shows that this smooth isotopy can be turned into a symplectic isotopy preserving $\mathcal{L}$ (observe that all the quantities appearing in the argument can be chosen to vanish over a neighborhood of $\mathcal{L}$ ).

A final remark about the homotopy class of the sections we construct in the non simply connected case : the homotopy class of $\left(s_{k}\right)_{\mid \mathcal{L}}$ as given by our construction is in fact related to the evaluation of $\omega$ on elements of $\pi_{2}(X, \mathcal{L})$. More precisely, given a loop $\gamma \subset \mathcal{L}$ bounding a disc $D$ in $X$, the trivialization of $L^{\otimes k}$ over $\gamma$ which minimizes the norm of the connection 1-form differs from the one which extends over $D$ by an amount of twisting approximately equal to $\frac{1}{2 \pi} \int_{D} k \omega$; therefore, in the construction of $W_{k}$ we obtain a linking number differing from this amount by at most a bounded quantity.

3.4. Behavior of concentrated sections along normal slices. For any point $x \in \mathcal{L}$, let $N_{x}$ be the image by the exponential map of the metric $g$ of a small disc in the normal space to $\mathcal{L}$ at $x$. Let $\sigma_{k, \mathcal{L}}$ be the asymptotically holomorphic sections concentrated over $\mathcal{L}$ constructed in $\S 2$. The following Lemma will be useful for applications.

Lemma 5. There exist constants $\delta>0$ and $\gamma>0$, independent of $k$, such that the restriction of $\left|\sigma_{k, \mathcal{L}}\right|^{2}$ to the intersection of $N_{x}$ with $B_{g_{k}}(x, \delta)$ is strictly concave, with second derivatives bounded from above by $-\gamma$ w.r.t. $g_{k}$, and reaches its maximum at a point within $g_{k}$-distance o(1) from $x$. The set of all these maxima is a smooth submanifold $\mathcal{L}_{k}^{\prime}, C^{0}$-converging towards $\mathcal{L}$ as $k$ increases. Moreover, when $X$ is Kähler the same properties remain true for the holomorphic sections $\tilde{\sigma}_{k, \mathcal{L}}$ constructed in $\S 3.1$.

Proof. Fix a value of $k$ and a point $p \in P_{k}$ such that $d_{g_{k}}(x, p) \leq k^{1 / 10}$, and work in the approximately holomorphic Darboux coordinate chart used to define $\sigma_{k, p}$; recalling that $\mathcal{L}$ is locally mapped to a linear subspace, let $N_{x}^{\prime}$ be the affine subspace through $x$ orthogonal to $\mathcal{L}$ in these coordinates. Since $x$ lies at $g$-distance less than $k^{-2 / 5}$ from $p$ where the coordinate map is an isometry, $N_{x}$ and $N_{x}^{\prime}$ are very close to each other (their angle at $x$ is at most $O\left(k^{-2 / 5}\right)$ ). Moreover, the restriction to $N_{x}^{\prime}$ of the function $f(z)=$ $\exp \left(-\frac{1}{4}|z|^{2}\right)$ is strictly concave (with a uniform upper bound on its second derivatives) and admits a maximum at $x$; therefore, $f_{\mid N_{x}}$ is also strictly concave and admits a maximum within $g$-distance $O\left(k^{-4 / 5}\right)$ from $x$. Since $\sigma_{k, p}$ coincides with $f^{k}$ near $x$, the same property holds for $\left|\sigma_{k, p}\right|^{2}$, except that the upper bound on second derivatives depends on $d_{g_{k}}(p, x)$ and only holds over a ball of fixed $g_{k}$-radius around $x$.

Next, recall from the proof of Lemma 4 that the contributions to $\sigma_{k, \mathcal{L}}$ coming from the various points of $P_{k}$ lying within $g_{k}$-distance $k^{1 / 10}$ from $x$ do not cancel each other at $x$, and more precisely their complex arguments 
at $x$ differ from each other by at most $O\left(k^{-2 / 5}\right)$. Of course this no longer remains true as soon as one moves away from $\mathcal{L}$; still, by a computation similar to the proof of Lemma 4 we can obtain control on the manner in which the complex arguments of the various contributions to $\sigma_{k, \mathcal{L}}$ differ from each other at a point close to $x$.

More precisely, consider a geodesic arc $\gamma$ joining $x$ to a nearby point $y$ in $N_{x}$, and let $p$ be a point of $P_{k}$ within $g_{k}$-distance $k^{1 / 10}$. Then

$$
\operatorname{Im} \int_{0}^{1}\left(\frac{\nabla \sigma_{k, p}}{\sigma_{k, p}}\right)_{\gamma(t)} \cdot \gamma^{\prime}(t) d t=\int_{0}^{1}-\frac{i k}{4} \sum z_{j} d \bar{z}_{j}-\bar{z}_{j} d z_{j} \cdot \gamma^{\prime}(t) d t
$$

is equal to $-\frac{k}{2} \omega_{0}(x-p, y-x)+O\left(k d_{g}(x, p)^{2} d_{g}(x, y)\right)$, where $\omega_{0}$ is the standard symplectic form on $\mathbb{C}^{n}$ and the error term comes from the nonlinearity of $N_{x}$ in the Darboux coordinate chart. In particular, if $p, p^{\prime}$ and $y$ are at bounded $g_{k}$-distance from $x$ then the difference of complex arguments between the contributions of $\sigma_{k, p}$ and $\sigma_{k, p^{\prime}}$ to $\sigma_{k, \mathcal{L}}(y)$ is given by $\phi_{p, p^{\prime}}(y)=\frac{k}{2} \omega_{0}\left(p-p^{\prime}, y-x\right)+O\left(k^{-2 / 5}\right)$, where the first term is bounded by a fixed constant times $d_{g_{k}}(y, x)$.

Fix a large constant $D>0$ (independent of $k$ and $x$ ), and let us first restrict ourselves to the sum $\sigma_{k, \mathcal{L}, x, D}$ of the contributions of the points of $P_{k}$ within $g_{k}$-distance $D$ from $x$. It follows from the above remarks that there exists a constant $\delta(D)>0$ (of the order of $D^{-1}$ ) such that $\left|\sigma_{k, \mathcal{L}, x, D}\right|^{2}$ is a strictly concave function at every point of $N_{x} \cap B_{g_{k}}(x, \delta(D))$, with a uniform upper bound (independent of $k, D$ and $x$ ) on its second derivatives. Indeed,

$$
\left|\sigma_{k, \mathcal{L}, x, D}(y)\right|^{2}=\sum_{p}\left|\sigma_{k, p}(y)\right|^{2}+\sum_{p \neq p^{\prime}}\left|\sigma_{k, p}(y)\right|\left|\sigma_{k, p^{\prime}}(y)\right| \cos \phi_{p, p^{\prime}}(y) .
$$

When $d_{g_{k}}(y, x)$ is not too large, $\cos \phi_{p, p^{\prime}}$ has second derivatives bounded from above by $o(1)$ (by the above expression of $\phi_{p, p^{\prime}}$ and the corresponding bounds on its first and second derivatives) ; therefore, using the lower bounds on $\left|\sigma_{k, p}\right|,\left|\sigma_{k, p^{\prime}}\right|$ and $\cos \phi_{p, p^{\prime}}$, the upper bounds on their second derivatives and the estimates on their first derivatives near $x$, we obtain that all the terms in the sum are strictly concave functions, thus yielding the desired concavity property for $\left|\sigma_{k, \mathcal{L}, x, D}\right|^{2}$.

Moreover, since the total contribution of the remaining points of $P_{k}$ to the section $\sigma_{k, \mathcal{L}}$ decreases exponentially fast as a function of $D$, it cannot affect the concavity property provided that $D$ is chosen large enough.

The contributions of the points within distance $k^{1 / 10}$ from $x$ reach their maxima over $N_{x}$ within $g$-distance $O\left(k^{-4 / 5}\right)$ from $x$ and their arguments at $x$ differ by $O\left(k^{-2 / 5}\right)$, while the remaining terms decrease exponentially fast with $k$. Therefore, the value of $\left|\sigma_{k, \mathcal{L}}(x)\right|^{2}$ is sufficiently close to the maximal possible one in order to guarantee that the maximum of $\left|\sigma_{k, \mathcal{L}}\right|^{2}$ over $N_{x}$ is reached within $g_{k}$-distance $o(1)$ from $x$.

Finally, the smoothness of the set $\mathcal{L}_{k}^{\prime}$ of all maxima is an immediate consequence of the smoothness of $\sigma_{k, \mathcal{L}}$ and of the uniform concavity property.

In the Kähler case, recall from $\S 3.1$ that the sections $\sigma_{k, p}$ are now constructed using the local holomorphic section $f(z)=1-\frac{1}{4}|z|^{2}+O\left(|z|^{3}\right)$, for which the maximum over $N_{x}^{\prime}$ is reached not necessarily at $x$ but at an arbitrary point within $g$-distance $O\left(k^{-4 / 5}\right)$ from $x$; however this does not 
affect the properties of $\left|\sigma_{k, p}\right|_{\mid N_{x}}^{2}$ that we have used. Similarly, the fact that $f$ is no longer real-valued affects the complex arguments of the various contributions to $\sigma_{k, \mathcal{L}}$, both at a point $x \in \mathcal{L}$ (bound by $O\left(k^{-1 / 5}\right)$ instead of $O\left(k^{-2 / 5}\right)$ in Lemma 4 , see $\S 3.1$ ) and outside $\mathcal{L}$ (but it turns out that these extra contributions do not affect the estimates) ; still, the argument remains valid without modification. Finally, since the holomorphic section $\tilde{\sigma}_{k, \mathcal{L}}$ differs from $\sigma_{k, \mathcal{L}}$ by an amount decreasing exponentially fast with $k$, it enjoys the same concavity and maximum properties as $\sigma_{k, \mathcal{L}}$, so that the conclusion remains valid in this case as well.

Remark. The assertions of Lemma 5 are also trivially satisfied by the concentrated sections obtained in the alternate proof of Theorem 2 outlined at the end of $\S 2$.

3.5. Relations with Lagrange skeleta. Let $X$ be a compact Kähler manifold, let $s$ be a holomorphic section of $L^{\otimes k}$, transverse to 0 , and consider the smooth hypersurface $W=s^{-1}(0)$. It is a result of Biran [B] that the section $s$ determines a splitting $X=B \sqcup \Delta$, where $B$ is a "standard" symplectic disc bundle over $W$ and $\Delta$ is an isotropic CW-complex called the Lagrange skeleton of $(X, W)$. The skeleton $\Delta$ is obtained as the union of the ascending varieties of all the critical points of the plurisubharmonic function $\log |s|^{2}$; it is well-known that these critical points are all of index at least $n$. Combined with standard results in Lagrangian intersection theory, this result provides powerful restrictions on Lagrangian embeddings. For example, any simply connected embedded Lagrangian submanifold in $X$ must intersect either $W$ or $\Delta$ (otherwise it could be disjointed from itself by a Hamiltonian flow in $B-W)$.

Biran's result is generally expected to remain valid in the more general case of a symplectic manifold and a symplectic hypersurface "of Donaldson type". However, to be on the safe side we will assume throughout this section that $X$ is Kähler, considering only the construction of $\S 3.1$.

Proposition 2. Let $\mathcal{L}$ be a compact isotropic submanifold of $X$. Then for large $k$ there exist holomorphic sections $s_{k}$ of $L^{\otimes k}$, transverse to 0 and nonvanishing over $\mathcal{L}$, such that $\mathcal{L}$ is contained in arbitrarily small neighborhoods of the Lagrange skeleta $\Delta_{k}$ corresponding to their zero sets $W_{k}$.

Proof. We use the notations of $\S 3.1$, and consider the local behavior near $\mathcal{L}$ of the transverse sections $s_{k}$ constructed as small perturbations of the concentrated holomorphic sections $\tilde{\sigma}_{k, \mathcal{L}}$. By Lemma 5 we know that the restriction of $\left|\tilde{\sigma}_{k, \mathcal{L}}\right|^{2}$ to each normal slice $N_{x}$ is locally concave and reaches its maximum close to $\mathcal{L}$. Therefore, choosing the transverse sections $s_{k}$ close enough to $\tilde{\sigma}_{k, \mathcal{L}}$ we conclude that the restriction of $h_{k}=\log \left|s_{k}\right|^{2}$ to $N_{x}$ admits a unique local maximum at $g_{k}$-distance less than $\frac{1}{2} \delta$ from $x$; as in Lemma 5 , the set of these local maxima is a smooth submanifold $\mathcal{L}_{k}^{\prime \prime}$ in $X$, obtained from $\mathcal{L}$ by an arbitrarily small deformation.

Observe that, by construction, every critical point of $h_{k \mid \mathcal{L}_{k}^{\prime \prime}}$ is also a critical point of $h_{k}$, with index increased by $\operatorname{codim} \mathcal{L}$. Moreover, although the union $\Lambda_{k}$ of the ascending varieties of these critical points is not exactly $\mathcal{L}_{k}^{\prime \prime}$, one expects it to be a small deformation of $\mathcal{L}$ as well. More precisely, observe 
that the gradient of $h_{k}$ is directed inwards at every point of the boundary of the $\delta$-tubular neighborhood $T_{\delta}(\mathcal{L})$ of $\mathcal{L}$ (w.r.t. $g_{k}$ ). This implies, first, that every point of $\Lambda_{k}$ lies at $g_{k}$-distance less than $\delta$ from $\mathcal{L}$, since all ascending trajectories remain in $T_{\delta}(\mathcal{L})$. Conversely, consider the disc $D_{x}=N_{x} \cap$ $B_{g_{k}}(x, \delta)$ and its image by the downward gradient flow of $h_{k}$ : since no trajectory can re-enter $T_{\delta}(\mathcal{L})$, the algebraic intersection number of the disc with $\mathcal{L}_{k}^{\prime \prime}$ constantly remains equal to 1 , which implies that $D_{x} \cap \Lambda_{k}$ is nonempty. In particular $\mathcal{L}$ is contained in the $\delta$-neighborhood of $\Lambda_{k}$, which is itself contained in the Lagrange skeleton.

3.6. Obstructions to Lagrangian embeddings. In this section, we no longer assume that $X$ is Kähler, but we assume that $\mathcal{L}$ is Lagrangian (i.e., $\operatorname{dim} \mathcal{L}=n$ ). It was suggested to us by Seidel, Viterbo and Biran that Theorem 2 might provide obstructions to the existence of certain Lagrangian embeddings by arguing along the following lines.

Consider the asymptotically holomorphic sections $s_{k}$ of $L^{\otimes k}$, bounded from below over $\mathcal{L}$ and uniformly transverse to 0 , given by Theorem 2 , and their zero sets $W_{k}$. It follows from Lemma 5 that, if the sections constructed in $\S 2$ are chosen sufficiently close to the concentrated sections $\sigma_{k, \mathcal{L}}$, their norms reach local maxima over the transverse slices $N_{x}$ along smooth submanifolds $\mathcal{L}_{k}^{\prime \prime}$ obtained by slightly deforming $\mathcal{L}$. Moreover, after an arbitrarily small perturbation we can assume that $h_{k}=\log \left|s_{k}\right|^{2}$ is a generic Morse function over $X-W_{k}$, without affecting the other properties.

Consider a point $x \in \mathcal{L}_{k}^{\prime \prime}$ where the restriction of $h_{k}$ to $\mathcal{L}_{k}^{\prime \prime}$ reaches a local minimum : it is a critical point of index $n$ of $h_{k}$. However the sections $s_{k}$ are asymptotically holomorphic and uniformly transverse to 0 , so it follows from a result of Donaldson [D1] that the critical points $h_{k}$ are all of index at least $n$. Therefore, the genericity condition on $h_{k}$ implies that the stable manifold $\Delta_{x}$ is a topological disc in $X-W_{k}$, with boundary mapped to $W_{k}$, and intersecting $\mathcal{L}_{k}^{\prime \prime}$ transversely at $x$. Observe that $\Delta_{x}$ is the image by the downward gradient flow of $h_{k}$ of the small disc $\Delta_{x} \cap T_{\delta}(\mathcal{L})$, where $T_{\delta}(\mathcal{L})$ is the $\delta$-tubular neighborhood of $\mathcal{L}$. However, the downward gradient flow is pointing outwards at every point of the boundary of $T_{\delta}(\mathcal{L})$, so that $x$ is the only intersection between $\mathcal{L}_{k}^{\prime \prime}$ and $\Delta_{x}$, and the intersection pairing between these two cycles evaluates to 1 . This implies that the homology class $\left[\mathcal{L}_{k}^{\prime \prime}\right] \in H_{n}\left(X-W_{k}\right)$ is a primitive element. Since $\mathcal{L}_{k}^{\prime \prime}$ is isotopic to $\mathcal{L}$, we obtain the following

Proposition 3. The element $[\mathcal{L}] \in H_{n}\left(X-W_{k}\right)$ is primitive.

Moreover, when $\mathcal{L}$ is not connected we can apply the same argument to the minima of $h_{k}$ over each component individually, obtaining that the fundamental classes of the various components of $\mathcal{L}$ are linearly independent primitive classes in $H_{n}\left(X-W_{k}\right)$.

When $X$ is a complex projective manifold, working with the holomorphic sections of $\S 3.1$ and assuming moreover that $\mathcal{L}$ is simply connected, it is an interesting question to ask whether the smooth complex hypersurfaces $W_{k}$ are always isotopic in $X-\mathcal{L}$ to hypersurfaces $H_{k}$ arbitrarily close to a given hyperplane section $H$ of $X$ avoiding $\mathcal{L}$. A positive answer would imply that 
$[\mathcal{L}]$ is primitive in $H_{n}(X-H)$ as well, providing a new proof of a theorem of Gromov.

However, even though no problem with homotopy classes of sections over $\mathcal{L}$ is to be feared in the simply connected case, the isotopy result of $\S 3.3$ does not apply in this context, as we have no control over the complex argument of the holomorphic section of $L^{\otimes k}$ defining $H_{k}$. Whether a refinement of Proposition 1 can handle this case or not remains an open question.

\section{REFERENCES}

[A1] D. Auroux, Asymptotically holomorphic families of symplectic submanifolds, Geom. Funct. Anal. 7 (1997), 971-995.

[A2] D. Auroux, Symplectic 4-manifolds as branched coverings of $\mathbb{C P}^{2}$, Invent. Math. 139 (2000), 551-602.

[B] P. Biran, Lagrangian barriers and symplectic embeddings, preprint.

[BT] R. Bott, L.W. Tu, Differential Forms in Algebraic Topology, Springer-Verlag, 1982.

[D1] S.K. Donaldson, Symplectic submanifolds and almost-complex geometry, J. Differential Geom. 44 (1996), 666-705.

[D2] S.K. Donaldson, Lefschetz pencils on symplectic manifolds, preprint.

[Du] J. Duval, Convexité rationnelle des tores lagrangiens de $\mathbb{C}^{2}$, C. R. Acad. Sci. Paris Sér. I Math. 307 (1988), 943-948.

[DS] J. Duval, N. Sibony, Polynomial convexity, rational convexity, and currents, Duke Math. J. 79 (1995), 487-513.

[Ga] D. Gayet, Ph.D. thesis, Université Toulouse III, in preparation.

[Gu] V. Guedj, Approximation of currents on complex manifolds, Math. Ann. 313 (1999), 437-474.

[McS] D. McDuff, D. Salamon, Introduction to Symplectic Topology, Oxford Mathematical Monographs, 1995.

[M] J.-P. Mohsen, Ph.D. thesis, ENS Lyon, in preparation.

[S] J.-C. Sikorav, Construction de sous-variétés symplectiques (d'après S. K. Donaldson et D. Auroux), Séminaire Bourbaki. Vol. 1997/98. Astérisque 252 (1998), 231-253.

[W] A. Weinstein, Symplectic manifolds and their Lagrangian submanifolds, Advances in Math. 6 (1971), 329-346.

Department of Mathematics, M.I.T., Cambridge MA 02139-4307, USA

E-mail address: auroux@math.mit.edu

Laboratoire Emile Picard, Université Paul Sabatier, 118 route de NarBonne, 31062 Toulouse Cedex 4, France

E-mail address: gayet@picard.ups-tlse.fr

UMPA, ENS-Lyon, UMR 5669, 46 allée d'Italie, 69364 Lyon Cedex 7, France

E-mail address: jpmohsen@umpa.ens-lyon.fr 